\title{
The Darboux-Bäcklund transformation for the static 2-dimensional continuum Heisenberg chain
}

\author{
Jan L. Cieśliński* \\ Joanna Czarnecka \\ Uniwersytet w Białymstoku, Instytut Fizyki Teoretycznej \\ ul. Lipowa 41, 15-424 Białystok, Poland
}

August 13, 2018

\begin{abstract}
We construct the Darboux-Bäcklund transformation for the sigma model describing static configurations of the 2-dimensional classical continuum Heisenberg chain. The transformation is characterized by a non-trivial normalization matrix depending on the background solution. In order to obtain the transformation we use a new, more general, spectral problem.
\end{abstract}

PACS Numbers: 02.30.Ik, 03.50.Kk, 05.45Yv.

Keywords: Integrable systems, sigma models, Darboux-Bäcklund transformation, 2-dimensional Heisenberg ferromagnet, Darboux matrix, dressing method.

\section{Introduction}

In this paper we consider 2-dimensional Euclidean $O(3) \sigma$-model

$$
n,{ }_{x x}+n,_{y y}+(n, \underset{x}{2}+n, \underset{y}{2}) n=0, \quad n^{2}=1,
$$

${ }^{*}$ E-mail: janek @alpha.uwb.edu.pl 
where $n \in \mathbb{E}^{3}$. This equation appears in the classical field theory and solid state physics. Considering $2+1$-dimensional $S^{2} \sigma$-model [10]

$$
\partial^{\mu} \partial_{\mu} \phi+\left(\partial^{\mu} \phi \cdot \partial_{\mu} \phi\right) \phi, \quad \phi \cdot \phi=0
$$

and 2+1-dimensional continuum classical Heisenberg ferromagnet equation

$$
\vec{S}_{, t}=\vec{S} \times\left(\vec{S}_{x x}+\vec{S}_{y y}\right), \quad \vec{S}^{2}=1,
$$

we see that their static solutions satisfy (11).

This $\sigma$-model plays important role also in differential geometry. The normal vector $n$ to surfaces of constant mean curvature endowed with conformal coordinates satisfies (11) 3, 8,

There are many interesting papers on the interpretation of the sigma model (11) and on the construction of special solutions [1, 3, 4, 9, 11]. In this paper we present a large family of gauge-equivalent spectral problems associated with the $\sigma$-model (11) and construct the Darboux-Bäcklund transformation for this general spectral problem.

\section{The spectral problem}

We consider the spectral problem of the form

$$
\begin{aligned}
& \Psi, x=U \Psi \equiv\left(A \zeta-\frac{A^{\dagger}}{\zeta}+R\right) \Psi \\
& \Psi,_{y}=V \Psi \equiv\left(B \zeta-\frac{B^{\dagger}}{\zeta}+S\right) \Psi,
\end{aligned}
$$

(where $U, V, \Psi$ are $2 \times 2$ matrices) uniquely characterized by the following properties:
(A) $U, V$ are rational in $\zeta$ with simple poles at $\zeta=0$ and $\zeta=\infty$,
(B) $\quad(U(1 / \zeta))^{\dagger}=-U(\bar{\zeta}), \quad(V(1 / \zeta))^{\dagger}=-V(\bar{\zeta})$
(C) $A^{2}=B^{2}=0$,
(D) $B=i A$. 
The constraint (B) implies $R^{\dagger}=-R, S^{\dagger}=-S$, i.e., $R, S$ are $u(2)$-valued. The compatibility conditions (the coefficient by $\lambda^{2}$ ) imply that $A$ and $B$ are parallel, i.e.,

$$
A=a W, \quad B=b W,
$$

where $a, b \in \mathbb{C}$. Without loss of the generality we can assume $a \in \mathbb{R}$ and

$$
a>0, \quad\left\langle W \mid W^{\dagger}\right\rangle=-2,
$$

where the scalar product on the space of $2 \times 2$ matrices is defined by $\langle X \mid Y\rangle=-2 \operatorname{Tr}(X Y)$. The coefficient 2 assures that the basis $\mathbf{e}_{k} \equiv-i \sigma_{k} / 2$ is orthonormal. We use the standard representation of Pauli matrices, i.e.,

$$
\mathbf{e}_{1}=\frac{1}{2}\left(\begin{array}{cc}
0 & -i \\
-i & 0
\end{array}\right), \quad \mathbf{e}_{2}=\frac{1}{2}\left(\begin{array}{cc}
0 & -1 \\
1 & 0
\end{array}\right), \quad \mathbf{e}_{3}=\frac{1}{2}\left(\begin{array}{cc}
-i & 0 \\
0 & i
\end{array}\right)
$$

The assumptions (6) make the choice of $W$ in equations (5) unique. Indeed, $\left\langle A \mid A^{\dagger}\right\rangle=|a|^{2}\left\langle W \mid W^{\dagger}\right\rangle=-2|a|^{2}$. Therefore $|a|^{2}=-\left\langle A \mid A^{\dagger}\right\rangle / 2=\operatorname{Tr}\left(A A^{\dagger}\right)$ and, finally,

$$
a=\sqrt{\operatorname{Tr}\left(A A^{\dagger}\right)}, \quad W=A / a .
$$

The constraint (D) reduces to $b=i a$ and is necessary to obtain the standard form of the Laplace operator (geometrically it means that we choose conformal coordinates on the corresponding constant mean curvature surface).

It is convenient to define the following frame

$$
E_{1}=\frac{W+W^{\dagger}}{2 i}, \quad E_{2}=\frac{W^{\dagger}-W}{2}, \quad E_{3}=\left[E_{1}, E_{2}\right] .
$$

Note that $\left\langle E_{k} \mid E_{j}\right\rangle=\delta_{k j}, E_{k}^{\dagger}=-E_{k}$ and $\operatorname{Tr} E_{k}=0$ for $k, j=1,2,3$. Thus this is an orthonormal basis in $s u(2)$. Any orthonormal basis in $s u(2)$ can be parameterized by a wector $W$, satisfying $W^{2}=0$ and $\operatorname{Tr}\left(W W^{\dagger}\right)=1$, according to the formulas (9).

The kinematics of the frame $E_{1}, E_{2}, E_{3}$ can be expressed in terms of six functions $\left(\alpha_{1}, \beta_{1}, \gamma_{1}, \alpha_{2}, \beta_{2}, \gamma_{2}\right)$ :

$$
\begin{aligned}
\frac{\partial}{\partial x}\left(\begin{array}{c}
E_{1} \\
E_{2} \\
E_{3}
\end{array}\right) & =\left(\begin{array}{ccc}
0 & \alpha_{1} & \beta_{1} \\
-\alpha_{1} & 0 & \gamma_{1} \\
-\beta_{1} & -\gamma_{1} & 0
\end{array}\right)\left(\begin{array}{l}
E_{1} \\
E_{2} \\
E_{3}
\end{array}\right) \\
\frac{\partial}{\partial y}\left(\begin{array}{c}
E_{1} \\
E_{2} \\
E_{3}
\end{array}\right) & =\left(\begin{array}{ccc}
0 & \alpha_{2} & \beta_{2} \\
-\alpha_{2} & 0 & \gamma_{2} \\
-\beta_{2} & -\gamma_{2} & 0
\end{array}\right)\left(\begin{array}{l}
E_{1} \\
E_{2} \\
E_{3}
\end{array}\right)
\end{aligned}
$$


satisfying the compatibility conditions:

$$
\begin{aligned}
& \alpha_{1, y}-\alpha_{2,{ }_{x}}+\beta_{2} \gamma_{1}-\beta_{1} \gamma_{2}=0, \\
& \beta_{1, y}-\beta_{2,{ }_{x}}+\alpha_{1} \gamma_{2}-\alpha_{2} \gamma_{1}=0, \\
& \gamma_{1, y}-\gamma_{2},{ }_{x}+\alpha_{2} \beta_{1}-\alpha_{1} \beta_{2}=0 .
\end{aligned}
$$

Denoting $\zeta=\exp (-i \kappa)$ and expressing $U, V$ in terms of $E_{k}$, we rewrite the spectral problem (4) as follows:

$$
\begin{aligned}
& U=a E_{2} \cos \kappa-a E_{1} \sin \kappa+R, \\
& V=a E_{2} \sin \kappa+a E_{1} \cos \kappa+S,
\end{aligned}
$$

The compatibility conditions for the spectral problem (12) read

$$
\begin{aligned}
& \left(a E_{2}\right),_{y}-\left(a E_{1}\right),_{x}+a\left[E_{2}, S\right]-a\left[E_{1}, R\right]=0, \\
& \left(a E_{2}\right),_{x}+\left(a E_{1}\right),,_{y}+a\left[E_{1}, S\right]+a\left[E_{2}, R\right]=0, \\
& R,,_{y}-S,_{x}+[R, S]=a^{2} E_{3} .
\end{aligned}
$$

The $u(2)$-valued functions $R$ and $S$ are linear combinations of $i I, E_{1}, E_{2}, E_{3}$ (with real coefficients), i.e., $S=i s_{0}+s_{1} E_{1}+s_{2} E_{2}+s_{3} E_{3}$ and $R=i r_{0}+$ $r_{1} E_{1}+r_{2} E_{2}+r_{3} E_{3}$. Thus the system (13) can be written in a more explicit form:

$$
\begin{aligned}
& r_{1}-\gamma_{1}=\beta_{2}+s_{2}, \quad r_{2}+\beta_{1}=\gamma_{2}-s_{1}, \\
& a,_{x}+a \alpha_{2}-a s_{3}=0, \quad a,,_{y}-a \alpha_{1}+a r_{3}=0, \quad r_{0}, y=s_{0}, x \\
& r_{1, y}-s_{1},,_{x}+r_{2} s_{3}-r_{3} s_{2}-\alpha_{2} r_{2}-\beta_{2} r_{3}+\alpha_{1} s_{2}+\beta_{1} s_{3}=0, \\
& r_{2, y}-s_{2}, x+r_{3} s_{1}-r_{1} s_{3}+\alpha_{2} r_{1}-\gamma_{2} r_{3}-\alpha_{1} s_{1}+\gamma_{1} s_{3}=0, \\
& r_{3, y}-s_{3, x}+r_{1} s_{2}-r_{2} s_{1}+\beta_{2} r_{1}+\gamma_{2} r_{2}-\beta_{1} s_{1}-\gamma_{1} s_{2}=4 a^{2} .
\end{aligned}
$$

Proposition 1. Let $\Psi$ satisfies (4), (A)-(D), and (9) holds. Then

$$
n=\Psi^{-1} E_{3} \Psi
$$

satisfies Eq. (11), i.e., $n_{x x}+n,_{y y}=f(x, y) n$, where $f$ is a real function. 
Proof: We compute $n,_{x x}+n,_{y y}$ and obtain, as a consequence, a linear combination of $\Psi^{-1} E_{k} \Psi(k=1,2,3)$. It is enough to show that the result is proportional to $\Psi^{-1} E_{3} \Psi$, i.e., that the coefficients by $\Psi^{-1} E_{1} \Psi$ and $\Psi^{-1} E_{2} \Psi$ vanish. The coefficient by $\Psi^{-1} E_{2} \Psi$ is given by

$$
\begin{aligned}
& \left(r_{1}-\gamma_{1}\right),_{x}-\left(\gamma_{2}-s_{1}\right),_{y}+\left(\beta_{1}+r_{2}\right)\left(r_{3}-\alpha_{1}\right)+\left(\beta_{2}+s_{2}\right)\left(s_{3}-\alpha_{2}\right) \\
& +\left(a,,_{y}-a \alpha_{1}+a r_{3}\right) \cos \kappa+\left(a,,_{x}+a \alpha_{2}-a s_{3}\right) \sin \kappa .
\end{aligned}
$$

To show that this expression vanish we use the first two equations of the system (14), then we eliminate all derivatives using appropriate equations of (11) and (14). Using once more (if necessary) the first equation of (14) we see that the obtained result is zero. The coefficient by $\Psi^{-1} E_{1} \Psi$

$$
\begin{aligned}
& -\left(r_{2}+\beta_{1}\right)_{, x}-\left(\beta_{2}+s_{2}\right)_{, y}+\left(s_{1}-\gamma_{2}\right)\left(s_{3}-\alpha_{2}\right)+\left(r_{3}-\alpha_{1}\right)\left(r_{1}-\gamma_{1}\right) \\
& -\left(a,,_{x}+a \alpha_{2}-a s_{3}\right) \cos \kappa-\left(a,,_{y}+a r_{3}-a \alpha_{1}\right) \sin \kappa
\end{aligned}
$$

vanishes as well what can be shown in exactly the same way.

Remark 1. The coefficients of the matrix $n$ with respect to the basis (7), $n=n_{1} \mathbf{e}_{1}+n_{2} \mathbf{e}_{2}+n_{3} \mathbf{e}_{3}$, identify this matrix with an $\mathbb{E}^{3}$ vector $\left(n_{1}, n_{2}, n_{3}\right)$. In other words, we use the isomorphism between su(2) and $\mathbb{E}^{3}$.

Remark 2. If one more constraint, namely $\operatorname{Tr} U=\operatorname{Tr} V=0$, $\operatorname{det} \Psi=1$, is imposed on the linear problem (4), then the Sym-Tafel formula $F=\Psi^{-1} \Psi_{,}$ yields surfaces of constant mean curvature, compare [6, 9].

\section{Gauge transformations}

The spectral problem (4) is invariant with respect to gauge transformations of the form $\hat{\Psi}=G \Psi$, where $G$ is any $\zeta$-independent $U(2)$-valued matrix $\left(G^{-1}=G^{\dagger}\right)$.

Proposition 2. If $\Psi$ satisfies (4), (A)-(D), and $\hat{\Psi}=G \Psi$, where $G^{-1}=G^{\dagger}$, then $\hat{\Psi}$ satisfies (4), (A)-(D) as well. Moreover

$$
\hat{n} \equiv \hat{\Psi}^{-1} \hat{E}_{3} \hat{\Psi}=n \text {. }
$$

Proof: $\quad \hat{\Psi}_{x_{x}}=\hat{U} \hat{\Psi}, \hat{\Psi}_{,_{y}}=\hat{V} \hat{\Psi}$, where

$$
\begin{aligned}
& \hat{U}=G A G^{-1} \zeta-\frac{G A^{\dagger} G^{-1}}{\zeta}+R+G,_{x} G^{-1} \equiv \hat{A} \zeta-\frac{\hat{A}^{\dagger}}{\zeta}+\hat{R}, \\
& \hat{V}=G B G^{-1} \zeta-\frac{G B^{\dagger} G^{-1}}{\zeta}+S+G,_{y} G^{-1} \equiv \hat{B} \zeta-\frac{\hat{B}^{\dagger}}{\zeta}+\hat{S},
\end{aligned}
$$


where $\left(G A G^{-1}\right)^{\dagger}=G A^{\dagger} G^{-1}$ because $G^{\dagger}=G^{-1}$. Obviously, $\hat{A}^{2}=\hat{B}^{2}=0$, $\hat{R}^{\dagger}=-\hat{R}, \hat{S}^{\dagger}=-\hat{S}$, etc. Thus the matrices $\hat{U}, \hat{V}$ satisfy all conditions (A)-(D). Then $\operatorname{Tr}\left(\hat{A} \hat{A}^{\dagger}\right)=\operatorname{Tr}\left(G A G^{-1} G A^{\dagger} G^{-1}\right)=\operatorname{Tr}\left(A A^{\dagger}\right)$. Hence, taking into account (8) and (9), we get $\hat{a}=a, \hat{W}=G W G^{-1}$ and $\hat{E}_{k}=G E_{k} G^{-1}(k=1,2,3)$. Finally, $\hat{n}=\Psi^{-1} G^{-1} G E_{3} G^{-1} G \Psi=n$.

Proposition 3. There exists a matrix $G=G(x, y) \in U(2)$ transforming the spectral problem (4), (A)-(D) into

$$
\begin{aligned}
& \hat{\Psi}_{,_{x}}=\left(a \mathbf{e}_{+} \zeta-\frac{a \mathbf{e}_{-}}{\zeta}+\hat{R}\right) \hat{\Psi}, \\
& \hat{\Psi}_{, y}=\left(i a \mathbf{e}_{+} \zeta+\frac{i a \mathbf{e}_{-}}{\zeta}+\hat{S}\right) \hat{\Psi},
\end{aligned}
$$

where $a$ is given by (8) and

$$
\mathbf{e}_{+}=\left(\begin{array}{cc}
0 & 1 \\
0 & 0
\end{array}\right), \quad \mathbf{e}_{-}=\left(\begin{array}{ll}
0 & 0 \\
1 & 0
\end{array}\right) .
$$

Proof: Any two orthonormal bases in $\mathbb{E}^{3}$ are related by an orthogonal transformation, which in turn can be represented by a unitary matrix (the spinor representation). In particular, the basis $E_{1}, E_{2}, E_{3}$ from Section 2 can be obtained from any constant orthonormal basis $\mathbf{e}_{1}, \mathbf{e}_{2}, \mathbf{e}_{3}$ by the transformation of the form $E_{k}=G^{-1} \mathbf{e}_{k} G, G \in U(2)$ (or even $G \in S U(2)$, if both bases have the same orientation). Applying the gauge transformation $\hat{\Psi}=G \Psi$ to the spectral problem (4) we obtain (17), where $\mathbf{e}_{+}, \mathbf{e}_{-}$are constant matrices such that $\mathbf{e}_{-}=\mathbf{e}_{+}^{\dagger}, \mathbf{e}_{+}^{2}=\mathbf{e}_{-}^{2}=0$, $\left\langle\mathbf{e}_{+} \mid \mathbf{e}_{-}\right\rangle=-2$. If $\mathbf{e}_{k}$ are given by (7), then $\mathbf{e}_{ \pm}$are given by (18).

Remark 3. If $\hat{\Psi}$ solves (17), then $n=\hat{\Psi}^{-1} \mathbf{e}_{3} \hat{\Psi}$ satisfies (1).

Remark 4. The spectral problem (17) or its equivalents are usually applied in the spectral approach to constant mean curvature surfaces, see [3, [6, 9].

\section{The Darboux-Bäcklund transformation}

Our aim is to construct the transformation $\tilde{\Psi}=D \Psi$ (where $D$ depends on $x, y$ and $\zeta$ ) in such a way that $\tilde{U}=D,{ }_{x} D^{-1}+D U D^{-1}$ and $\tilde{V}=$ $D, y D^{-1}+D V D^{-1}$ have the same form as $U, V$ (compare [5]). In other 
words, the properties (A), (B), (C), (D) of Section 2 should be preserved by the transformation. We confine ourselves to the simplest case

$$
D=\mathcal{N}\left(I+\frac{\zeta_{1}-\mu_{1}}{\zeta-\zeta_{1}} P\right)
$$

where the matrices $\mathcal{N}$ and $P$ do not depend on $\zeta, P^{2}=P$, and $\zeta_{1}, \mu_{1}$ are complex parameters $\left(\zeta_{1} \neq \mu_{1}\right)$.

The property (A) implies, by virtue of a well known result of Zakharov and Shabat [12,

$$
\operatorname{ker} P \ni \Psi\left(\zeta_{1}\right) \vec{b}, \quad \operatorname{im} P \ni \Psi\left(\mu_{1}\right) \vec{c},
$$

where $\vec{b}, \vec{c} \in \mathbb{C}^{2}$ are constant vectors and $\zeta_{1}, \mu_{1} \in \mathbb{C}$ are constant as well.

One can easily check that the property $(\mathrm{B})$ is preserved if $D^{-1}(\bar{\zeta})=$ $D^{\dagger}(1 / \zeta)$ which yields, after straightforward computations,

$$
P^{\dagger}=P, \quad \bar{\mu}_{1}=\frac{1}{\zeta_{1}}, \quad \mathcal{N N}^{\dagger}=1+\left(\left|\zeta_{1}\right|^{2}-1\right) P
$$

Therefore the condition $\mu_{1} \neq \zeta_{1}$ is equivalent to $\left|\zeta_{1}\right| \neq 1$. Moreover, $P^{\dagger}=P$ implies $\vec{c}_{1} \perp \vec{b}_{1}$. P is explicitly expressed by the matrix $\Psi\left(1 / \vec{\zeta}_{1}\right)$ :

$$
P=\frac{1}{1+|\xi|^{2}}\left(\begin{array}{cc}
|\xi|^{2} & \xi \\
\bar{\xi} & 1
\end{array}\right)
$$

where $\xi=u_{1} / u_{2}$ and $\left(u_{1}, u_{2}\right)^{T}=\Psi\left(1 / \bar{\zeta}_{1}\right) \vec{c}$. One can check that the equation $\mathcal{N} \mathcal{N}^{\dagger}=1+\left(\left|\zeta_{1}\right|^{2}-1\right) P$ is satisfied by

$$
\mathcal{N}=\mathcal{N}_{0}\left(I+\left(\zeta_{1} e^{i \sigma}-1\right) P\right)
$$

where $\mathcal{N}_{0}$ is a unitary matrix $\left(\mathcal{N}_{0}^{-1}=\mathcal{N}_{0}^{\dagger}\right)$ and $\sigma$ is a real constant.

Considering the spectral problem (17) we have to take into account one more constraint: $W=\mathbf{e}_{+}$is a fixed constant matrix, given for instance by (18). In this case

$$
\tilde{a} \mathbf{e}_{+}=a \mathcal{N} \mathbf{e}_{+} \mathcal{N}^{-1}
$$

and from (24) we can compute $\mathcal{N}_{0}$.

In the following we focus on the more general spectral problem (4) and the matrix $\mathcal{N}_{0}$ can be arbitrary. Actually, the matrix $\mathcal{N}_{0}$ is not important as far as the transformation of $n$ is concerned (compare Proposition 21). 
Without loss of the generality we will assume $\mathcal{N}_{0}=I$. Finally we arrive at following formula for the Darboux matrix:

$$
D=\left(I+\left(\zeta_{1} e^{i \sigma}-1\right) P\right)\left(I+\frac{\zeta_{1}-\bar{\zeta}_{1}^{-1}}{\zeta-\zeta_{1}} P\right)=I+\left(e^{2 i \beta}-1\right) P
$$

where

$$
e^{2 i \beta}:=\frac{\left(\zeta_{1}-\zeta\left|\zeta_{1}\right|^{2}\right) e^{i \sigma_{1}}}{\left|\zeta_{1}\right|^{2}-\zeta \bar{\zeta}_{1}}
$$

Note that $\beta$ is real (because $\bar{\zeta}=\zeta^{-1}$ ) and $\beta$ does not depend on $x, y$.

One can always parameterize the Hermitean projector $P$ by a unit vector $\vec{p}=\left(p_{1}, p_{2}, p_{3}\right)$ :

$$
P=\frac{1}{2}(I+\mathbf{p}), \quad \mathbf{p}:=\sum_{k=1}^{3} p_{k} \sigma_{k}, \quad p_{1}^{2}+p_{2}^{2}+p_{3}^{2}=1 .
$$

The function $\xi$ appearing in (22) is a stereographic projection of $\vec{p}$ :

$$
\xi=\frac{p_{1}-i p_{2}}{1-p_{3}}, \quad p_{1}=\frac{2 \operatorname{Re} \xi}{1+|\xi|^{2}}, \quad p_{2}=\frac{-2 \operatorname{Im} \xi}{1+|\xi|^{2}}, \quad p_{3}=\frac{|\xi|^{2}-1}{|\xi|^{2}+1} .
$$

The spectral problem (17) can be considered as a particular case of (4) and any solution $\Psi$ of (17) satisfies (4) as well, compare Remark 3 Therefore we can take as a background solution

$$
\mathbf{n}=\Psi^{-1} \mathbf{e}_{3} \Psi
$$

where $\Psi$ is a solution of (17). According to Remark 1 we associate with $\mathbf{n}$ a unit vector $\vec{n}:=\left(n_{1}, n_{2}, n_{3}\right)$ defined by:

$$
\mathbf{n}=\sum_{k=1}^{3} n_{k} \mathbf{e}_{k}
$$

The Darboux-Bäcklund transformation of $\mathbf{n}$ yields:

$$
\tilde{\mathbf{n}}=\Psi^{-1} D^{-1} \mathbf{e}_{3} D \Psi .
$$

The obtained expression can be computed as follows

$$
D^{-1} \mathbf{e}_{3} D=\frac{1}{2 i}(\cos \beta-i \mathbf{p} \sin \beta) \sigma_{3}(\cos \beta+i \mathbf{p} \sin \beta),
$$

and simplified in a straightforward way:

$$
D^{-1} \mathbf{e}_{3} D=\frac{1}{2 i}\left(\sigma_{3} \cos 2 \beta+2 p_{3} \mathbf{p} \sin ^{2} \beta+\left(p_{2} \sigma_{1}-p_{1} \sigma_{2}\right) \sin 2 \beta\right) .
$$




\section{Special solutions}

We will compute explicitly the action of the Darboux-Bäcklund transformation on a simple background. The simplest seed solution can be obtained from the requirement $U=$ const, $V=$ const and $\Psi$ satisfies (17). Then $E_{k}=\mathbf{e}_{k}$ are constant (i.e., $\alpha_{k}=\beta_{k}=\gamma_{k}=0$ ) and $a=a_{0}=$ const. Thus the system (14) reduces to

$$
r_{1}=s_{2}, \quad r_{2}=-s_{1}, \quad s_{3}=r_{3}=0, \quad r_{1} s_{2}-r_{2} s_{1}=a_{0}^{2},
$$

and can be easily solved:

$$
s_{1}=-r_{2}=a_{0} \cos \delta_{0}, \quad r_{1}=s_{2}=a_{0} \sin \delta_{0},
$$

where $\delta_{0}$ is an arbitrary real parameter. Therefore,

$$
\begin{aligned}
& U=a_{0} \mathbf{e}_{2}\left(\cos \kappa-\cos \delta_{0}\right)-a_{0} \mathbf{e}_{1}\left(\sin \kappa-\sin \delta_{0}\right), \\
& V=a_{0} \mathbf{e}_{1}\left(\cos \kappa+\cos \delta_{0}\right)+a_{0} \mathbf{e}_{2}\left(\sin \kappa+\sin \delta_{0}\right),
\end{aligned}
$$

and, finally

$$
\begin{aligned}
& U=2 a_{0} \sin \delta_{-}\left(\mathbf{e}_{1} \cos \delta_{+}+\mathbf{e}_{2} \sin \delta_{+}\right), \\
& V=2 a_{0} \cos \delta_{-}\left(\mathbf{e}_{1} \cos \delta_{+}+\mathbf{e}_{2} \sin \delta_{+}\right),
\end{aligned}
$$

where $\delta_{ \pm}:=\frac{1}{2}\left(\delta_{0} \pm \kappa\right)$. Without loss of the generality we put $\delta_{0}=0$ (more general choice corresponds to symmetries of the sigma model (11) like rotation in the space of parameters $x, y$ and the $O(3)$ symmetry). Then

$$
-2 \mathbf{e}_{1} \cos \delta_{+}-2 \mathbf{e}_{2} \sin \delta_{+}=i\left(\begin{array}{cc}
0 & e^{-i \kappa / 2} \\
e^{i \kappa / 2} & 0
\end{array}\right)=: E .
$$

Note that $E^{2}=-1$. Therefore, if $U, V$ are constant, then the solution of the linear problem (4) is simply given by

$$
\Psi=\exp (x U+y V) C_{0}=\exp (\theta E) C_{0}=(\cos \theta+E \sin \theta) C_{0},
$$

where $C_{0}$ is a constant unitary matrix and

$$
\theta=\theta(x, y, \zeta)=a_{0} x \sin \frac{\kappa}{2}-a_{0} y \cos \frac{\kappa}{2} .
$$

Thus, taking into account $\zeta=e^{-i \kappa}$,

$$
\Psi(x, y, \zeta)=\left(\begin{array}{cc}
\cos \theta & i \sqrt{\zeta} \sin \theta \\
i \sin \theta / \sqrt{\zeta} & \cos \theta
\end{array}\right) C_{0} .
$$


Finally, using (29), we get the following background solution:

$$
n=\mathbf{e}_{1} \sin 2 \theta \sin \frac{\kappa}{2}-\mathbf{e}_{2} \sin 2 \theta \cos \frac{\kappa}{2}+\mathbf{e}_{3} \cos 2 \theta .
$$

Now, we will perform the Darboux-Bäcklund transformation. In order to compute $\xi$ we evaluate $\Psi$ at $\zeta=1 / \bar{\zeta}_{1}$ and denote $\lambda_{1}:=1 / \sqrt{\bar{\zeta}_{1}}$ :

$$
\Psi\left(x, y, \bar{\zeta}_{1}^{-1}\right)=\left(\begin{array}{cc}
\cos \theta_{1} & -i \lambda_{1} \sin \theta_{1} \\
-i \lambda_{1}^{-1} \sin \theta_{1} & \cos \theta_{1}
\end{array}\right) C_{0}
$$

where $\theta_{1}=\theta\left(x, y, \zeta_{1}^{-1}\right) \equiv P_{1}+i Q_{1}$, i.e.,

$$
\begin{aligned}
& P_{1}=-\frac{1}{2} a_{0}\left(1+\frac{1}{a_{1}^{2}+b_{1}^{2}}\right)\left(x b_{1}+y a_{1}\right), \\
& Q_{1}=\frac{1}{2} a_{0}\left(1-\frac{1}{a_{1}^{2}+b_{1}^{2}}\right)\left(x a_{1}-y b_{1}\right),
\end{aligned}
$$

where $a_{1}+i b_{1}:=\lambda_{1} \equiv \zeta_{1}^{-1 / 2}$ (we recall that by assumption $a_{1}^{2}+b_{1}^{2} \neq 1$ ). Then

$$
\xi=\frac{c_{1}-i c_{2} \lambda_{1} \tan \theta_{1}}{c_{2}-i c_{1} \lambda_{1}^{-1} \tan \theta_{1}}
$$

where $\left(c_{1}, c_{2}\right)^{T}=C_{0} \vec{c}$. Without loss of the generality we can put $c_{1}=0$ (one can show that more general choice is equivalent to the translation in the space of variabes $x, y$, compare [2]). Then, finally,

$$
\xi=\frac{\left(a_{1}+i b_{1}\right)\left(\sinh Q_{1} \cosh Q_{1}-i \sin P_{1} \cos P_{1}\right)}{\cosh ^{2} Q_{1} \cos ^{2} P_{1}+\sinh ^{2} Q_{1} \sin ^{2} P_{1}},
$$

where $P_{1}, Q_{1}$ are given by (43) and $a_{1}, b_{1}$ are arbitrary real parameters.

Therefore the solution $\tilde{\mathbf{n}}$ given by (31) can be easily computed using (32), (28), (40), (45) and (43), where $\beta, a_{0}, a_{1}, b_{1}, \kappa$ and the matrix $C_{0}$ are arbitrary constants. In particular, assuming $C_{0}=I$ and $\kappa=0$ we obtain $\tilde{\mathbf{n}}=\left(n_{1}, n_{2}, n_{3}\right)$, where

$$
\begin{aligned}
& n_{1}=2 p_{1} p_{3} \sin 2 \beta+p_{2} \sin 2 \beta, \\
& n_{2}=\left(2 p_{2} p_{3} \sin ^{2} \beta-p_{1} \sin 2 \beta\right) \cos 2 \theta-\left(\cos 2 \beta+2 p_{3}^{2} \sin ^{2} \beta\right) \sin 2 \theta, \\
& n_{3}=\left(2 p_{2} p_{3} \sin ^{2} \beta-p_{1} \sin 2 \beta\right) \sin 2 \theta+\left(\cos 2 \beta+2 p_{3}^{2} \sin ^{2} \beta\right) \cos 2 \theta .
\end{aligned}
$$

The functions $p_{1}, p_{2}, p_{3}$ are given by (28) and (45), $\theta$ is given by (39). 


\section{Conclusions}

In this paper we presented a new version of the Darboux-Bäcklund transformation for the sigma model (1). There are two interesting points in our construction. First, we introduced the spectral problem (4), more general than (17). Both spectral problems are gauge-equivalent and the sigma model (11) is invariant with respect to unitary gauge transformations of the spectral problem (compare Proposition 21). Second, the normalization matrix (23) is quite non-trivial. The matrix $\mathcal{N}$ depends on $x, y$ through the projector matrix $P$ (i.e., through the background wave function). Note that the Darboux-Bäcklund transformation for the spectral problem (17) is even more difficult. We have an additional constraint on the unitary matrix $\mathcal{N}_{0}$, namely (24), which is technically pretty complicated. From this point of view the spectral problem (4) is better.

Our approach is rather straightforward and we plan to generalize it for some related sigma models and geometric problems (surfaces of constant mean curvature in Euclidean and Lorentzian spaces). We hope also to extend this approach on higher dimensional problems using Clifford numbers (compare [7]).

\section{References}

[1] A.A.Belavin, A.M.Polyakov: JETP Lett. 22 (1975) 245.

[2] M.Błaszak: Multi-Hamiltonian theory of dynamical systems (Chapter 5), SpringerVerlag, Berlin 1998.

[3] A.I.Bobenko: "Surfaces in Terms of 2 by 2 Matrices. Old and New Integrable Cases", [in:] Harmonic maps and integrable systems (Aspects of Mathematics, vol. 23), pp. 83-128, edited by A.P.Fordy and J.C.Wood; Vieweg, Brunswick 1994.

[4] P.Bracken, A.M.Grundland, L.Martina: "The Weierstrass-Enneper system for constant mean curvature surfaces and the completely integrable sigma model", J.Math.Phys. 40 (1999) 3379-3403.

[5] J.Cieśliński: "An algebraic method to construct the Darboux matrix", J.Math.Phys. 36 (1995) 5670-5706.

[6] J.Cieśliński: "The Darboux-Bianchi-Bäcklund transformations and soliton surfaces", [in:] Nonlinearity $\&$ Geometry, pp. 81-107, edited by D. Wójcik and J. Cieśliński, PWN, Warsaw 1998.

[7] J.L.Cieśliński: "Geometry of submanifolds derived from Spin-valued spectral problems", Theor. Math. Phys. 137 (2003) 1394-1403. Translated from Teor. Matem. Fiz. 137 (2003) 47-58.

[8] J.Cieśliński, A.Sym: "Gauss map and Heisenerg ferromagnet", unpublished, presented at the conference Euromech 216, Leeds 1986.

[9] A.Doliwa, A.Sym: "Constant mean curvature surfaces in $E^{3}$ as an example of soliton surfaces", [in:] Nonlinear Evolution Equations and Dynamical Systems (Proceedings of the 7-th NEEDS conference, Baia Verde, Gallipoli (Italy), June 1991), pp. 111-117, edited by M.Boiti, L.Martina and L.Pempinelli; World Scientific, Singapore 1992. 
[10] R.A.Leese, M.Peyrard, W.J.Zakrzewski: Nonlinearity 3 (1990) 387.

[11] I.Sterling, H.C.Wente: "Existence and classification of constant mean curvature multibubbletons of finite and infinite type", Indiana Math. J. 42 (1993) 1239-1266.

[12] S.P.Novikov, S.V.Manakov, L.P.Pitaevskii, V.E.Zakharov: Theory of solitons, the inverse scattering method, Consultants Bureau, New York 1984 (translated from: V.E.Zakharov, S.V.Manakov, S.P.Novikov, L.P.Pitaevskii: Theory of solitons, Nauka, Moscow 1980). 